\title{
Self-reconstruction of single-cycle light bullet in linear (free-space) propagation regime
}

\author{
Sergey Chekalin ${ }^{1,}$, Victor Kompanets ${ }^{1}$, Alexander Dormidonov ${ }^{1}$, and Valerii Kandidov ${ }^{2,1}$ \\ ${ }^{1}$ Institute of Spectroscopy RAS, 108840 Moscow, Troitsk, Russia \\ ${ }^{2}$ Moscow Lomonosov State University, Physics Department, 119991 Moscow, Russia
}

\begin{abstract}
The results of investigation of extremely compressed wave packets penetration of air gap under single femtosecond laser pulse filamentation in $\mathrm{LiF}$ at anomalous group velocity dispersion are presented. It is revealed by laser coloration method that a single cycle light bullet formed before the gap up to $0.5 \mathrm{~mm}$ was recovered after passing some distance behind the gap which increased nonlinearly with the gap length.
\end{abstract}

The formation of light bullets (LBs) is the result of simultaneous and matched laser pulse self-compression both in space and time under conditions of anomalous group velocity dispersion [1, 2]. LB penetration of air gap in sapphire has been investigated in [3] and capability of LB to restore its parameters after the gap has been revealed. In the present work, the laser coloration technique [4] based on the long-lived structures of color centers (CCs) production by only one laser pulse is used to investigate this effect in LiF. In contrast to [3] laser coloration technique made it possible, first, to carry out the measurements without signal accumulation, i.e. using only one laser pulse, and second, without any inaccuracies that arise from the overlap of the radiations of supercontinuum, conical emission, and the plasma channel. The long-lived structures made up of CCs, may be easily detected and investigated under subsequent continuous laser illumination in their absorption band near 450 $\mathrm{nm}$. This technique allowed observation of the formation of single-cycle LBs and to reveal that it doesn't change its parameters after propagation through an induced waveguide $[2,5,6]$.

The experimental layout was similar to the one described in [6]. The $130 \mathrm{fs}$ pulse at $3200 \mathrm{~nm}$ with energy about $25 \mu \mathrm{J}$ was focused inside the LiF sample with air gap that can be changed from 25 to $500 \mu \mathrm{m}$ at a distance of several millimeters from its input face by thin lenses made of $\mathrm{CaF}_{2}$ with focal distance $97 \mathrm{~mm}$. To obtain a single-pulse exposure, the sample was translated after each shot in the direction perpendicular to the laser beam. To analyze the spatial distribution of the luminescence intensity of the recorded CCs we used methods of optical microscopy with illumination of the induced CCs by $450 \mathrm{~nm} \mathrm{cw}$ laser.

To analyze light field transformation during LB formation, we obtained the numerical solution of the unidirectional equation of mid-IR femtosecond pulse propagation in LiF [2]. As an initial condition we took a spectrally limited wave packet with a Gaussian distribution of the electric field amplitude in time and space corresponding to those used in experiment.

Longitudinal luminescence intensity profiles are presented in the left-hand side of Fig. 1. Calculated plasma density distributions in a channel during the propagation of a

\footnotetext{
* Corresponding author: schekalin@yandex.ru
} 
single-cycle LB at the same conditions (Fig. 1, right), are consistent with experimental measurement in spite of different nature of these signals, which manifested by difference in their envelopes. It can be seen that $25 \mu \mathrm{m}$ gap practically does not influence formation and propagation of LB in the sample. On the gap increasing the picture begin to change.
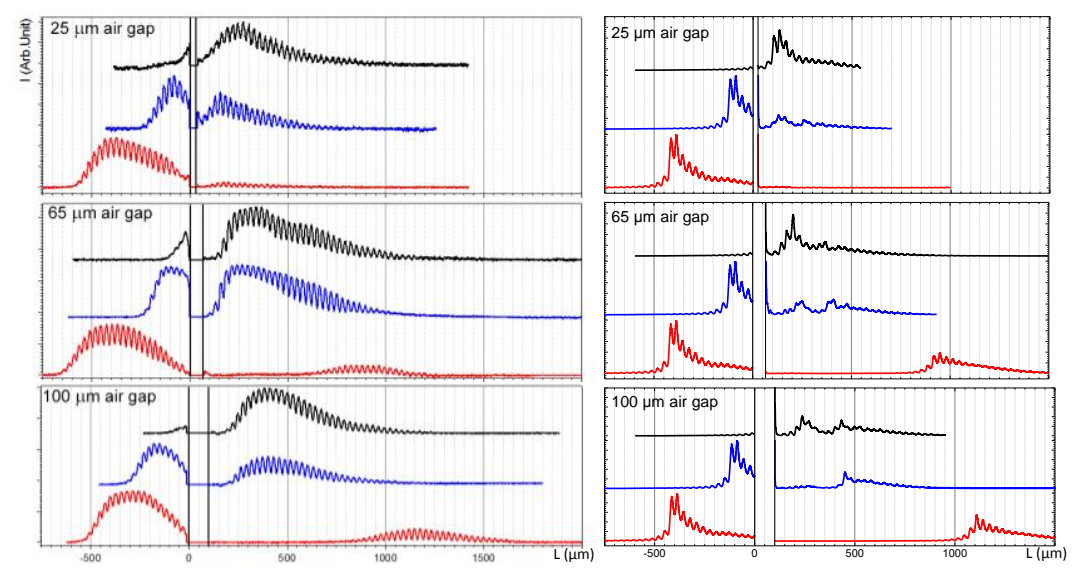

Fig. 1. Density profiles of the CCs recorded in experiments (left side). Calculated plasma density distributions in a channel during the propagation of a single-cycle LB at the same conditions (right side).

First, just after the gap a region with no luminescence arises followed by a region with periodic change of luminescence. Minimal length of this dip increases from $30 \mu \mathrm{m}$ at $65 \mu \mathrm{m}$ gap to $1300 \mu \mathrm{m}$ at $500 \mu \mathrm{m}$ gap. At that several times increasing behavior of this luminescence dip was observed when LB has already spent passing over the most of its pathway before the gap. It may be caused by the beam intensity decreasing after the gap due to divergence. Nevertheless the beam power keeps more than critical for self-focusing and it is spatially compressed under propagation after the gap attaining again the intensity sufficient for CC appearance. Numerical modeling shows that LB divergence at the output to air gap depends on the LB pathway before the gap. At that LB radiation always remains converging with focal length decreasing with this pathway. So, for instance, after $25 \mu \mathrm{m}$ air gap LB diameter does not increase, but it is not the case for $500 \mu \mathrm{m}$ gap when LB diverges after focusing in air. CC formation threshold appears to be about maximal intensity in $\mathrm{LB}\left(\sim 10^{14} \mathrm{~W} / \mathrm{cm}^{2}\right)$. Our estimations show that at our experimental parameters effective compression of LB [7] up to a single optical cycle takes place at all stages of its propagation in LiF before as well as after the air gap.

This work was supported by the Russian Science Foundation, project no. 18-12-00422.

\section{References}

1. Y. Silberberg, Opt. Lett. 22, 1282 (1990)

2. S. V. Chekalin et al., Physics - Uspekhi 62, 282 (2019)

3. I. Grazuleviciute et al., Optics Express 22, 30613 (2014)

4. S.V. Chekalin, V.O. Kompanets, Opt. Spectrosc. 127, 88 (2019)

5. S. V. Chekalin et al., Quantum Electron. 48, 372 (2018)

6. A. V. Kuznetsov et al., Quantum Electron. 46, 379 (2016).

7. E. D. Zaloznaya et al., Quantum Electron. 48, 366 (2018) 